Meta

Journal des traducteurs

Translators' Journal

\title{
L'enseignement de la traduction et de l'interprétation en Espagne
}

\section{Antonio Argüeso}

Volume 50, numéro 1, mars 2005

Enseignement de la traduction dans le monde

Teaching Translation Throughout the World

URI : https://id.erudit.org/iderudit/010670ar

DOI : https://doi.org/10.7202/010670ar

Aller au sommaire du numéro

Éditeur(s)

Les Presses de l'Université de Montréal

ISSN

0026-0452 (imprimé)

1492-1421 (numérique)

Découvrir la revue

Citer cet article

Argüeso, A. (2005). L'enseignement de la traduction et de l'interprétation en Espagne. Meta, 50(1), 223-230. https://doi.org/10.7202/010670ar
Résumé de l'article

Cet article fait le point sur les développements récents des études de traduction et d'interprétation en Espagne. L'analyse du nouveau cursus s'accompagne d'un passage en revue des publications scientifiques et des organisations professionnelles espagnoles en matière de traduction et d'interprétation. d'utilisation que vous pouvez consulter en ligne.

https://apropos.erudit.org/fr/usagers/politique-dutilisation/ 


\title{
L'enseignement de la traduction et de l'interprétation en Espagne
}

\author{
ANTONIO ARGÜESO \\ Haute École de Bruxelles, Bruxelles, Belgique \\ argueso@heb.be
}

\section{RÉSUMÉ}

Cet article fait le point sur les développements récents des études de traduction et d'interprétation en Espagne. L'analyse du nouveau cursus s'accompagne d'un passage en revue des publications scientifiques et des organisations professionnelles espagnoles en matière de traduction et d'interprétation.

\section{ABSTRACT}

This paper provides an update of studies in translation and interpretation in Spain. Not only does it analyze recent developments such as the new curriculum, it also reviews scientific publications and professional organizations connected to the field of translation and interpretation.

\section{MOTS-CLÉS/KEYWORDS}

Espagne, interprétation, formation, cursus, centres

\section{Introduction}

Créée en 1972, la Escuela Universitaria de Traductores e Intérpretes (EUTI) dépend de l'Universidad Autónoma de Barcelone et constitue le premier centre espagnol officiel destiné à la formation des traducteurs et des interprètes. Sept ans plus tard, l'Instituto de Idiomas de l'Universidad de Grenade se transforme en EUTI et l'Universidad de Las Palmas ouvre une nouvelle EUTI peu après; finalement, c'est à Malaga que s'ouvre la dernière EUTI juste avant que les études menant à la Licence en Traduction et Interprétation ne voient officiellement le jour en 1991.

L'année 1974 voit la création de l'Instituto Universitario de Lenguas Modernas y Traductores; il dépend de la Facultad de Filología de l'Universidad Complutense de Madrid et délivre un Master $^{1}$ en Traduction. Cet institut va déployer une grande activité dans l'organisation d'événements directement liés à ces études: cours spéciaux, journées et rencontres annuelles et autres formations post-universitaires. D'autre part, en 1988, La Laguna inaugure un Master en Interpretación de Conferencias. Il s'agit d'une formation orientée exclusivement vers la profession d'interprète de conférences; la notoriété de ce Master ne fera que croître au fil du temps.

Il convient de citer également le "Centro Universitario Cluny-Iseit», attaché à la Facultad de Letras del Instituto Católico de París et reconnu par la Comunidad de Madrid en octobre 1988. Les cours ont débuté en 1959, mais le diplôme n'a pas de valeur légale.

Nous parlons donc d'études de création tardive et, comme nous le verrons plus loin, les législateurs n'ont pas tenu compte en les organisant de l'expérience que la majorité des pays européens avaient déjà acquise dans ce domaine. 
Malgré tout, bien qu'arrivée tardivement sur la scène académique, cette filière va prospérer, rattraper son retard et corriger les défauts de la loi qui les régit.

\section{Licence en traduction et interprétation}

\subsection{La nouvelle filière}

En 1992, 5 universités ouvrent leurs portes aux étudiants désireux de suivre ces nouveaux cours, conformément au nouveau Plan de estudios de Traducción et Interpretación. Dix ans plus tard, elles seront 17.

Ce Plan de estudios offre des caractéristiques propres à l'idiosyncrasie académique espagnole:

1. Comme dans tout l'enseignement supérieur, cette formation ne se donne qu'à l'université; dans la majorité des cas, ces nouvelles études vont dépendre de facultés ou de départements déjà existants et, comme les études de Philologie et de Linguistique connaissent une baisse de fréquentation, beaucoup de centres vont se recycler et offriront des cours de traduction et d'interprétation, vu l'importance de la demande dans le nouveau domaine.

2. La loi limite le choix des matières: on ne peut, en principe, suivre que 6 matières différentes à la fois, et pas plus de 9 par an.

3. Le diplôme porte la mention de «traducción e interpretación», le législateur suppose donc une étroite corrélation dans la formation des deux professions.

4. Les matières sont divisées en : troncales (matières imposées à toutes les universités par le Ministère); obligatorias (imposées par chaque université); optativas (imposées par chaque faculté) et enfin de libre configuración (choisies librement par l'étudiant) - voir point 3. Les cours sont organisés en "crédits»: un total de 300, ce qui représente une moyenne de 25 heures de cours par semaine.

5. La décision d'accorder aux stages ou au travail de fin d'études une valeur d'heures de cours est laissée à l'appréciation de chaque centre.

6. La division des langues du décret est la suivante: A (langue active), B (langue vers laquelle l'on traduit), C1 (langue à partir de laquelle on traduit) et C2 (troisième langue étrangère).

7. L'accès direct au second cycle d'études est ouvert à tout diplômé universitaire sous certaines conditions.

\subsection{La formation générale du nouveau Plan de estudios}

Il va de soi que ces nouvelles études ont suscité maints commentaires, et les critiques de toutes sortes n'ont pas manqué de surgir de partout (force est de constater que, curieusement, personne n'a revendiqué la paternité du Plan de Estudios): des personnes chargées de la formation des étudiants et des responsables des centres d'enseignement déjà existants en Espagne qui ne furent pas consultés, des professionnels appartenant à des organismes européens qui, eux, furent bien consultés, mais dont les suggestions n'ont pas été retenues, etc.

Mais la filière a, malgré tout, fait du chemin et plus de 2500 jeunes sont inscrits dans ces disciplines : 500 diplômés en sortent chaque année. En fait, la véritable organisation des études varie considérablement d'une université à une autre :

1. Les universités ont le droit d'opérer une sélection des étudiants désireux d'entreprendre ce type d'études et d'exiger qu'ils aient déjà une bonne connaissance d'au moins une langue étrangère. Mais il faut noter que dans pratiquement toutes les universités les critères de sélection sont chaque année moins stricts. 
2. Seules quelques-unes des anciennes EUTIs disposent de professeurs spécialisés dans le domaine qui nous occupe. La plupart des universités qui ont organisé ces études ont dû le faire avec un corps professoral majoritairement issu des anciennes facultés de philologie et de linguistique (voir 3.4), ce qui ne garantit pas une formation adaptée aux besoins de la profession.

3. Les cours d'interprétation sont obligatoires pour tous les étudiants et le surpeuplement des auditoriums nuit à la qualité de l'enseignement.

4. L'offre de langues est, quant à elle, fort limitée (anglais, français et allemand) et seules deux anciennes EUTIs, celle de Barcelone (Universidad Autónoma) et celle de Grenade ${ }^{2}$ offrent un éventail beaucoup plus important de langues: arabe, chinois, italien, portugais, japonais et russe.

5. Le poids des cours d'interprétation est trop lourd pour ceux qui désirent se consacrer uniquement à la traduction.

\section{La formation}

\subsection{Matières à option et libre choix}

L'université européenne en général, et l'espagnole en particulier ${ }^{3}$, est confrontée à un nombre démesuré d'études, de filières et de spécialisations diverses.

En Espagne, comme par ailleurs dans toute l'Europe, les universités essaient de maîtriser leurs dépenses. C'est la raison pour laquelle elles tentent de regrouper les matières à option et de libre choix en obligeant des étudiants de diverses filières à les suivre ensemble. Dans le domaine qui nous occupe, les élèves de traduction suivront entre 30 et $50 \%$ de leurs cours avec des étudiants d'autres facultés (selon la politique d'économie de l'université ou l'influence du responsable des études de traduction/ interprétation au sein de l'université). Bien menée, cette politique pourrait aider les étudiants à se spécialiser et à choisir un domaine de traduction/interprétation particulier, mais comme les matières sont généralement pensées pour les destinataires «naturels» des cours (droit pour les étudiants de droit, médecine pour les futurs médecins...), les futurs traducteurs ont peu l'occasion d'appliquer ces cours à leur besoins.

\subsection{Matières dépendant d'autres départements}

Le lien entre matière et départements constitue un autre problème propre à la structure de l'université espagnole. N'entrons pas dans une analyse du système compliqué d'attribution entre centres, départements et aires de connaissance. Disons pour ceux qui ne sont pas familiarisés avec ce système, que la partie enseignement et recherche des départements espagnols recouvre toute l'université et que c'est à ces derniers qu'appartient le contrôle des programmes. De telle sorte qu'en traduction par exemple:

- la documentation dépend du département de Biblioteconomía

- l'informatique appliquée à la traduction, de celui des Ciencias de la Computación ou de celui des Lenguajes y sistemas informáticos

- les cours de langues dépendent du département de Filología ou de Lingüística correspondants

- la théorie et la pratique de la traduction dépend du département de Lingüística general

Il arrive souvent qu'un professeur consacre sa charge d'enseignant et de chercheur dans le domaine de sa propre licence et accessoirement en traduction/interprétation ${ }^{4}$; on 
comprend aisément qu'il lui soit difficile de s'adapter aux exigences particulières des étudiants.

Au moment de préparer les programmes d'études, c'est parfois plus en fonction des intérêts particuliers d'un département (ou d'un professeur) que l'on a introduit des matières et non en pensant à la formation des étudiants. Ceci explique l'apparition de matières étranges comme par exemple ce «cours de géographie médiévale» figurant pendant quelque temps au programme des études de traduction/interprétation dans une université espagnole.

\subsection{La formation des interprètes}

Accorder à tous les étudiants le titre de licencié en traduction et interprétation constitue une difficulté énorme au moment de mettre sur pied une formation conforme aux nécessités du marché, et cela surtout en interprétation. Et ce double titre justifie aussi les multiples critiques que cet enseignement s'attire de la part de divers secteurs de la société.

Ce manque de formation spécifique aux interprètes peut expliquer le succès persistant du Master en Interpretación de Conferencias octroyé par La laguna depuis 1988, alors que l'université ne décerne pas cette licence. D’autres universités essaient avec plus ou moins de bonheur d'organiser ces mêmes études post-universitaires (voir 5.2.1: les formations spécifiques offertes par chaque université dans ce domaine).

\subsection{Les professeurs de traduction et d'interprétation}

La création des études de traduction et d'interprétation est encore récente, et ce n'est que depuis peu qu'il est possible d'accéder au doctorat dans ce domaine. Peu de personnes réunissent en Espagne la double qualité d'enseignant spécialisé dans ce domaine et les titres nécessaires à l'enseignement universitaire.

Les universités qui organisent ces études ont dû, durant leurs premières années de fonctionnement, aller chercher des professeurs formés à cette spécialisation dans d'autres pays, ou avoir recours à d'anciens diplômés en traduction et interprétation ayant acquis une formation supplémentaire à la fin de leurs études, et surtout à des philologues recyclés ou en voie de recyclage; ces derniers n'ont quasiment jamais eu de contacts préalables avec la traduction ou l'interprétation (ou l'expérience qu'ils en ont est purement littéraire, une orientation qui n'est pas envisagée dans la formation qui nous occupe). Il faut sans doute voir dans ce que nous venons de dire l'explication du nombre démesuré de congrès, journées, rencontres, séminaires... sur la traduction et l'interprétation organisés continuellement en Espagne. Aucun autre pays ne réalise autant d'efforts dans la «formation» et la "passation» de ce savoir au moyen de ce genre de réunions.

\section{La connaissance préalable de langues étrangères des étudiants}

On envisage en Espagne l'obligation pour les élèves de secondaire d'étudier une langue étrangère et la possibilité d'en choisir une seconde; mais l'enseignement des langues n'ayant jamais connu dans notre pays un grand succès, le niveau des jeunes en langue étrangère n'est pas suffisant pour aborder ce genre d'études supérieures. 
La loi permet aux centres d'enseignement de la traduction/interprétation de faire passer des tests de connaissances aux futurs étudiants. L'intérêt pour ce type d'études est tel actuellement que plusieurs universités publiques se servent de cette opportunité pour sélectionner leurs étudiants. Mais lorsque la tendance de la demande de ces études sera renversée (c'est déjà le cas dans certaines régions), ces universités publiques se verront confrontées aux mêmes problèmes que les universités privées et, devant le manque d'étudiants, elles devront revoir leurs exigences à la baisse. Ceci va, sans aucun doute, provoquer une baisse du niveau de formation de ces professionnels, et l'on verra comme dans le domaine de l'interprétation de conférences apparaître des formations de troisième cycle visant à pallier les déficiences de la formation de deuxième cycle.

\section{Centres où l'on donne ces cours en Espagne Centres décernant le titre officiel de licencié}

\section{Centres publics}

1. Universitat Antònoma de Barcelona Facultat de Traducció i d'Interpretació (ancienne EUTI) <http://www.fti.uab.es/_fti_secretaria/> :

- Doctor en Traducció;

- Posgraus de traducció literaria; jurídica; audiovisual; tradumàtica;

- Course in translation and intercultural studies;

- DESE en Traducció;

- Màster en Traducció i Interpretació.

2. Universidad de Granada - Faculté de traduction et d'interprétation (ancienne EUTI) $<$ www.ugr.es $>$;

- Doctorats en traduction, société et communication dans les processus de traduction et d'interprétation;

- Spécialiste (expert) universitaire en interprétation du langage (espagnol) des signes.

3. Universidad de Las Palmas de Gran Canaria (ancienne EUTI) Faculté de traduction et d'interprétation $<$ http://www5.ulp.c.es/servidores/fti/index.htm $>$

4. Universidad de Málaga, Faculté de philosophie et lettres (ancienne EUTI) <www.uma.es>

5. Universitat Pompeu Fabra (1992) - Facultat de Traducció i Interpretació

$<$ http://www.upf.es/factii/index.htm>

- Programa de doctorat «La Traducció: Aspectes Literaris i Discursius»;

- Diplomas de postgrau "Interpretació de Conferència Mèdica»; "Tractament informàtic de la llengua»; "Anàlisi del Discurs i Pràctiques Socials»; "Traducció i Tractament Informàtic».

6. Universidad de Salamanca (1993) - Faculté de traduction et documentation, <http:// exlibris.usal.es>:

- Doctorat en traduction et interprétation: la traduction et les langages spécialisés.

7. Universitat Jaume I (1994) - Faculté des sciences humaines et sociales -, $<$ http://sic.uji.es/uji/acad/trad/trad.html $>$.

8. Universidad de Vigo (1992) - Facultad de Filoloxía e Traducción $<$ http://www.uvigo.es/centros/index.gl.htm>.

9. Universidad de Valladolid (1996) - Campus de Soria - Faculté de traduction et interprétation, $<$ http://www.uva.es/consultas/titulaciones.php?centro $=216 \&$ campus $=4>$

10. Universidad de Alicante (1996) - Faculté de philosophie et lettres $<$ http://www.ua.es/centros/filosofia/titulaciones.html>.

11. Centre d'études supérieures Philippe II (2000) - dépendant de l'université Complutense $<$ www.cesfelipesegundo.com $>$. 
12. Universidad del País Vasco Faculté de philologie, géographie et histoire (2000), <http: //www.vc.ehu.es/filologia/>.

13. Universidad Autónoma de Madrid (2002) - Faculté de philosophie et lettres (2002), $<$ http://www.uam.es/centros/filoyletras/licenciaturas/ltraduccion/traduccion.html >.

\section{Centres privés officiels}

1. Universidad Pontificia de Comillas (1993) - Faculté des sciences humaines et sociales, $<$ www.upco.es>. (Il s'agit d'une université de l'Église: en fin d'études, les étudiants doivent passer une épreuve d'homologation devant un jury de professeurs des universités publiques.) :

- Master en interprétation de conférences

2. Universidad Alfonso X El Sabio (1994) - Faculté de langues appliquées, <www.uax.es>

3. Universidad Europea de Madrid (1995) - Área de Comunicación - Facultad de Traducción e Interpretación <www.uem.es $>$.

4. Universitat de Vic (1995) - Facultat de Ciències Humanes, Traducció i Documentació $<$ http://www.uvic.es/fchtd/llicenciatura.html>

- Creació de materials amb suport multimèdia per a l'ensenyament de la Traducció.

\section{Autres centres}

1. Instituto Universitario de Lenguas Modernas y Traductores (IULMyT) de la Universidad Complutense de Madrid (1974) <www.ucm.es/info/iulmyt $>$ :

- Formation du troisième cycle dans le domaine de la traduction;

- Master en traduction.

2. Universidad de León <http://www.unileon.es/estructura/com_doc/programas/089. htm>:

- Doctorat en traduction interculturelle (1993).

3. Universidad de La laguna $<$ http://www.ull.es/master/mic/> :

- Master en interprétation de conférences (1988);

- Spécialisation universitaire en interprétation de langues (Expert universitaire) (2000).

4. L'École de traducteurs de la Universidad de Castilla-La Mancha (1994), <http:// www.uclm.es/traductor/ $>$ : une formation hétérogène centrée sur la culture méditerranéenne. On y donne des cours de traduction (littéraire, juridique, économique... à partir de l'arabe et surtout de l'hébreu).

5. Le Centre universitaire Cluny - I.S.E.I.T http://www.cluny-es.com lequel offre, en plus du titre en traduction et interprétation:

- Master en interprétation de conférences;

- Master en traduction dans la société de l'information multilingue.

\section{Revues et comptes-rendus périodiques sur la traduction en Espagne}

- Actas de los Encuentros Complutenses en torno a la Traducción, Madrid, Editorial Complutense. Il s'agit indubitablement des réunions les plus importantes organisées sur ce sujet en Europe et leurs comptes-rendus contiennent des articles signés par les principaux auteurs et professionnels dans ce domaine. On a publié les comptes-rendus des congrès de 1988, 1990, 1992, 1994, 1995 et 1997.

- Hieronymus Complutensis. Institut universitaire de langues modernes et de traduction, Édition de la Complutense (depuis 1995).

- Sendebar, bulletin de la faculté de traducteurs de l'Université de Grenade (depuis 1990).

- Senez, Rrevue de l'association des traducteurs basques - Paseo de la Zurriola 14 - 1 E20002 Donostia (existe depuis 1978). 
- Quaderns de Traducció i Interpretació (1982-1992 y 1998). Publiés d'abord par la EUTI et la faculté de Traduction de l'Université Autónoma de Barcelone.

- Livius, revue d'études de traduction. Université de Leon (depuis 1992).

- Viceversa. Revista Galega de Traducción. Publiée par ATG - Association des traducteurs galiciens et le département de Filoloxía Galega da Universidade de Vigo (desde 1995).

- Trans. Revue de traductologie de l'Université de Malaga (depuis 1966).

- Vasos comunicantes, revue de l'Association des traducteurs littéraires. Sagasta, $285^{\circ} \mathrm{A}$ 28004 Madrid.

\section{Les associations de traducteurs et interprètes}

ACE-Traducteurs - http://www.acett.org/ - Section autonome des traducteurs littéraires de l'association du Collège des écrivains: association très active. Elle publie la revue Vasos comunicantes.

APETI - http://www.terminometro.info/b28/es/apeti.htm - Association nationale des traducteurs, laquelle a connu de graves problèmes internes et ne représente pour l'instant qu'une partie des professionnels.

ATIC - http://www.atic.cc/ - Association des traducteurs et interprètes catalans.

ATG - http://www.uvigo.es/webs/webatg/home.html - Association des traducteurs galiciens; publie la revue Viceversa.

EIZIE - http://www.eizie.org/english/home_en.htm - Association des traducteurs basques; publie la revue Senez.

TRIAC - http://www.teclata.es/triac/espanish/triac.html - Traductors i Interprets Associat Pro-Collegi.

AIIC - http://www.espaiic.net/espaiic/index.html - Association internationale d'interprètes de conférences; représentée en Espagne.

AICE - http://www.aice-interpretes.com/ - Association espagnole des interprètes de conférences, plus représentative au niveau national que la précédente.

\section{NOTES}

1. Le titre de «Master» n'est pas réglementé en Espagne.

2. L'université de Comillas offre également l'italien et le japonais comme langues $\mathrm{C}$.

3. Nous l'avons déjà dit, en Espagne, pratiquement toutes les études supérieures sont organisées à l'université, depuis le tourisme jusqu'à l'informatique, en passant par le sport, les sciences optiques, la kinésithérapie, etc.

4. De fait, le législateur «a oublié» de créer une aire de connaissances spécifique à la «traduction/ interprétation ». L'association de Centros de Formación de Traducción e Interpretación del Estado Español dut livrer une dure bataille afin d'arriver à la création de l'aire en question.

\section{RÉFÉRENCES}

Actas del departament de Traducció i d'Interpretació (1997) : Universitat Autònoma de Barcelona, Actas del «I Congrés Internacional sobre traducció (1992)» y Actas del «II Congrés Internacional sobre traducció (1994)», 716 p.

Argüeso, A. (1997) : «Formación de traductores en España» Senez 19, p. 127-135.

Arranz, J. C. (1991): «Estudios de Licenciatura de Traducción Técnica en la Universidad de Hildesheim», Sendebar 2, p. 155-166.

Bueno García, A. (1999) : «La formación del traductor y del intérprete: retos y perspectivas » in Traducción, Interpretación y Lenguaje, en Antonio ARgüEso (ed.) Traducción, Interpretación y Lenguaje, Expolingua, Madrid, p. 81-87.

Gallardo San Salvador, N. y D. Sánchez (eds.) (1992): La enseñanza de la terminología. Actas del Coloquio Iberoamericano sobre enseñanza de la terminología, Granada, I.C.E. Universidad. 
Gallardo, N., Kelly, D., Rivera, L. y C. Seisbel (1988) : «La reforma de los planes de estudios para la formación de traductores y el mercado del trabajo: comentarios generales sobre la situación de su enseñanza en España. Jornadas Europeas de Traducción e Interpretación. Universidad de Granada - EUTI, p. 93-96.

Jornadas sobre diseño curricular del traductor e intérprete (1996): Facultad de Traducción e Interpretación de la Universidad de Granada, 344 p.

Libro blanco de la traducción en España (1997): ACE-Traductores, Madrid, 204 p.

SAntoyo, J. C. (1996) : «Bibliografía de la traducción», Universidad de León.

Tricás Preckler, M. (1998): «Traducción e Interpretación: El plan de estudios y los objetivos de formación» Hieronymus Complutensis, nº 8, p. 99-106.

Le site Internet de l'Institut Cervantes offre une page intéressante sur la traduction: <http:// cvc.cervantes.es/obref/aproximaciones/>. 